\title{
Supraventricular tachycardias in the first year of life: what is the best pharmacological treatment? 24 years of experience in a single centre
}

\author{
Guglielmo Capponi ${ }^{1}$, Gilda Belli ${ }^{1}$, Mattia Giovannini ${ }^{1}{ }^{0}$, Giulia Remaschi ${ }^{2}$, Alice Brambilla ${ }^{3}$, \\ Francesca Vannuccini ${ }^{3}$, Silvia Favilli ${ }^{3}$, Giulio Porcedda ${ }^{3}$ and Luciano De Simone ${ }^{3^{*}}$ (1)
}

\begin{abstract}
Background: Supraventricular tachycardias (SVTs) are common in the first year of life and may be life-threatening. Acute cardioversion is usually effective, with both pharmacological and non-pharmacological procedures. However, as yet no international consensus exists concerning the best drug required for a stable conversion to sinus rhythm (maintenance treatment). Our study intends to describe the experience of a single centre with maintenance drug treatment of both re-entry and automatic SVTs in the first year of life.
\end{abstract}

Methods: From March 1995 to April 2019, 55 patients under one year of age with SVT were observed in our Centre. The SVTs were divided into two groups: 45 re-entry and 10 automatic tachycardias. As regards maintenance therapy, in re-entry tachycardias, we chose to start with oral flecainide and in case of relapses switched to combined treatment with beta-blockers or digoxin. In automatic tachycardias we first administered a beta-blocker, later combined with flecainide or amiodarone when ineffective.

Results: The patients' median follow-up time was 35 months. In re-entry tachycardias, flecainide was effective as monotherapy in $23 / 45$ patients $(51.1 \%$ ) and in 20/45 patients (44.4\%) in combination with nadolol, sotalol or digoxin (overall 95.5\%). In automatic tachycardias, a beta-blocker alone was effective in 3/10 patients (30.0\%), however, the best results were obtained when combined with flecainide: overall 9/10 (90\%).

Conclusions: In this retrospective study on pharmacological treatment of SVTs under 1 year of age the combination of flecainide and beta-blockers was highly effective in long-term maintenance of sinus rhythm in both re-entry and automatic tachycardias.

Keywords: Supraventricular tachycardia, Infant, Flecainide, Beta-blockers

*Correspondence: I.desimone@meyer.it

${ }^{3}$ Cardiology Unit, Anna Meyer Children's University Hospital, Viale

Gaetano Pieraccini 24, 50139 Florence, Italy

Full list of author information is available at the end of the article

\section{Background}

Supraventricular tachycardias (SVTs) are the most common arrhythmia occurring in the first year of life, with an incidence of $1 / 250-1 / 1000$ live births and $1 / 10$ in patients with congenital heart diseases [1]. In these patients, the aetiology of SVT must be sought in immaturity of the conduction system and a major sensitivity to catecholamines $[2,3]$. In recent years the management 
of the prevention of supraventricular tachycardia recurrences has shifted towards the use of Class III antiarrhythmic drugs (sotalol and amiodarone) or Class IC drugs (flecainide and propafenone), with success rates comparable with digoxin and beta-blocking agents. However, the "gold standard" therapy in terms of safety and efficacy for long-term treatment has not been established [4]. Our retrospective study evaluated the different antiarrhythmic response of re-entry and automatic tachycardias to maintenance therapy mainly with Class IC drugs and beta-blockers, used either alone or in combination.

\section{Methods}

We performed an observational retrospective study among patients suffering from SVTs occurring in the first year of life and referred to the Cardiology Unit of the Anna Meyer Children's University Hospital in Florence from March 1995 to April 2019. All the patients were treated in the Intensive Care Unit and data were collected by selecting and reviewing paper-based medical records, and since January 2000, electronic medical records. The patients' inclusion criteria were an SVT at onset between the fetal period and the end of the first year of life. We divided the patients into two sub-groups: re-entry or automatic tachycardias. Since they have the same mechanism, we included atrioventricular re-entry tachycardias (AVRT), atrioventricular nodal re-entry tachycardias (AVNRT), and paroxysmal junctional reciprocating tachycardias (PJRT) in the re-entry tachycardias. These different arrythmias were diagnosed on the basis of 12-lead ECGs (trans-thoracic or trans-oesophageal) according to the European Society of Cardiology practice guidelines and other criteria [5-7]: (a) in an AVRT, the P wave is usually identifiable in the ascending or descending branch of the $T$ wave, the PR is longer than the RP' inter$\mathrm{val}$, the $\mathrm{T}$ wave follows about $70 \mathrm{~ms}$ after the QRS; (b) in an AVNRT, the $\mathrm{P}$ wave is identifiable as a pseudo $\mathrm{r} 1$ wave in V1 and a pseudo s wave in inferior leads; (c) a PJRT is characterized by an $\mathrm{R}-\mathrm{P}$ ' interval longer than $\mathrm{P}-\mathrm{R}$ with the $\mathrm{P}-\mathrm{R}$ interval being normal or short, negative $\mathrm{P}$ waves in leads II, III and aVF and the absence of "warm up" at SVT onset; (d) automatic tachycardia is recognized by a progressive shortening in the $\mathrm{P}-\mathrm{P}$ interval at the onset of SVT and an R-P' interval longer than the P-R interval.

We also used intravenous adenosine as an additional diagnostic test: in re-entry forms it abruptly restores the sinus rhythm, whereas it only reduces the ventricular rate in most automatic forms [8]. All the patients were treated with acute therapy, defined as treatments used with the aim of restoring the sinus rhythm: adenosine, intravenous or oral flecainide, intravenous amiodarone or intravenous propafenone, and non-pharmacological treatments (diving reflex, trans-oesophageal stimulation or direct current shock). We used flecainide as a firstline drug in re-entries, whereas we decided to start with a beta-blocker in the automatic forms $[9,10]$. In case of unresponsiveness, other drugs were added. The short and long-term efficacy of flecainide and beta-blockers was assessed. Flecainide was administered orally at a dose ranging from 50 to $120 \mathrm{mg} / \mathrm{m}^{2} /$ day and when the sinus rhythm was not stable for more than 3 half-lives, we decided to add a beta-blocker (sotalol $0.5-2.5 \mathrm{mg} / \mathrm{kg} /$ day, propranolol 1-3 mg/kg/day, nadolol $1-5 \mathrm{mg} / \mathrm{kg} /$ day) or digoxin $0.005-0.0075 \mathrm{mg} / \mathrm{kg} /$ day. We used amiodarone, $75-250 \mathrm{mg} / \mathrm{m}^{2} /$ day, as a third-line agent $[4,11,12]$. Digoxin has been replaced by beta-blockers since 2000 . In automatic tachycardias we decided to start with beta blockers (propranolol, nadolol or sotalol) at the above dosage schedule. When a stable sinus rhythm was not achieved after 3 half-lives, we decided to add flecainide and, in case of unresponsiveness, shifted to the combination with amiodarone. Since October 2014 the serum concentration of flecainide has been assessed in all patients by liquid chromatography tandem-mass spectrometry and it has been kept in a range of $200-800 \mathrm{ng} /$ $\mathrm{ml}$. Measuring the drug concentration helped us to identify under or overdosage and allowed for changing the oral therapy, irrespective of clinical symptoms or ECG alterations. We also reported the time to sustained sinus rhythm, defined as the days between the first administration of an antiarrhythmic agent and achievement of the stable sinus rhythm. After discharge, each patient had home monitoring for 1 year at the most, and they all underwent a regular follow-up in our Cardiology Unit where we documented any supraventricular tachycardia relapses, therapeutic variations or adverse effects. In the absence of recurrences during the previous 6 months or after the first year of life with at least one week without antiarrhythmic therapy, we performed a trans-oesophageal study. When we were not able to induce a re-entry arrhythmia, the drug therapy was discontinued. The statistical analysis can be divided into two parts: for one we used descriptive values as mean, standard deviation (SD), median and discrete values as percentage using Excel algorithms; for the other we used the t-test to compare time to cardioversion in re-entry and automatic tachycardias.

\section{Results \\ Study population}

From March 1995 to April 2019, 55 patients with SVTs occurring in the first year of life were monitored at our centre and satisfied our criteria. 6/55 patients (10.9\%) had congenital heart disease (CHD): 3 ventricular septal defects, 1 ventricular septal defect associated with atrial septal defect, 1 non-compacted left ventricular 
cardiomyopathy associated with ventricular septal defect and 1 Ebstein anomaly. In 7/55 patients (12.7\%), the arrhythmia occurred during fetal life. Median postnatal age at onset was 12.5 days (1-303 days, mean 40.9 days \pm 77.6 days SD). Of these, $45 / 55$ (81.2\%) had a re-entry tachycardia (Table 1): 40/45 (88.8\%) had AVRT, 4/45 (8.8\%) AVNRT, and 1/45 (2.2\%) a PJRT. The patients' heart rate ranged between 200 and $340 \mathrm{bpm}$. The clinical symptoms at onset were documented in $43 / 45$ patients (95.5\%): 2/43 (4.6\%) had cardiogenic shock, 19/43 (44.1\%) symptoms related to heart failure (dyspnoea, failure to thrive, pallor, drowsiness), and 22/43 (51.1\%) minor symptoms (restlessness, persistent crying). In 10/55 patients (18.1\%), the ECG detected automatic tachycardias (Table 2); heart rate ranged between 180 and $300 \mathrm{bpm}$. Clinical presentation was cardiogenic shock in $1 / 10$ patients $(10.0 \%)$, heart failure-related symptoms in $2 / 10(20.0 \%)$, and minor symptoms in 7/10 (70.0\%).

\section{Responses}

During the median 35 months' follow-up (from 0 to 289 months), we obtained different responses in re-entry and automatic tachycardias (Fig. 1). In re-entry tachycardias, flecainide was effective as monotherapy in 23/45 patients $(51.1 \%)$, while in $20 / 45$ (44.4\%), a stable cardioversion was achieved after adding digoxin in $7 / 20$ patients (35.0\%), sotalol in $3 / 20(15.0 \%)$, nadolol in $8 / 20(40.0 \%)$, and propranolol in $2 / 20(10.0 \%)$. For $2 / 45$ patients $(4.4 \%)$, flecainide was ineffective: in both cases the amiodarone plus nadolol combination was effective. As regards automatic tachycardias, the beta-blockers nadolol, propranolol and sotalol in monotherapy were effective in $3 / 10$ patients $(30.0 \%)$. The others, $(7 / 10)(70.0 \%)$, required the combination of beta-blockers with another drug: flecainide in $6 / 7(85.7 \%)$ cases, and amiodarone in $1 / 7$ (14.2\%). In the majority of patients, flecainide was effective with two or three administrations per day, in $2 / 52$ cases (3.8\%), however, the number of doses had to be increased: one patient was given six doses of flecainide and four of nadolol a day and the other patient was stabilized with six doses of flecainide and three of sotalol a day. Unlike re-entry supraventricular tachycardias, stable cardioversion was delayed in automatic tachycardias. In re-entry tachycardias, we obtained sustained sinus rhythm between 1 and 22 days, while in automatic tachycardias we achieved the same result between 6 and 40 days $(p<0.05)$ (Table 3$)$. In all but one case, 1/55 (QTc equal to $500 \mathrm{~ms}$ ), the QTc interval was in the normal range. At the last follow-up (median 35 months), $29 / 55(52.7 \%)$ patients were still in maintenance therapy, while $19 / 55$ (34.5\%) had stopped their drug therapy in accordance with the trans-oesophageal study. 5/55 (9.0\%) patients underwent radiofrequency ablation at $7,10,12$
(2 patients) and 13 years of age. Maintenance therapy had been carried out before discontinuation for a median of 16 months (from 10 to 72 months). We had six hospital readmissions: three patients received flecainide and nadolol, two flecainide and sotalol, and one propafenone; stable maintenance of sinus rhythm was obtained thanks to dose increments and in one patient by shifting to amiodarone and nadolol.

\section{Safety}

One patient died during follow-up because of decompensated heart failure related to glycogenosis type 2 . Flecainide caused significant adverse events in 2/52 (3.8\%) patients: one had severe bradycardia $(80 \mathrm{rpm})$ with wide QRS and atrioventricular dissociation, transitory pallor, and hypotonia, followed by rapid and complete recovery after discontinuing treatment. Another death involved an outpatient unsuccessfully treated with intravenous flecainide elsewhere, who, after administration of the correct oral dose of flecainide, experienced severe intraventricular conduction disorders, bradycardia, ventricular arrhythmias, evolving into asystole and cardiac arrest, unresponsive to cardiopulmonary resuscitation treatment. Both adverse events were related to accidental overdosage: in the first, the flecainide concentration was 2 times higher, and in the second, 6 times higher than normal. Two other patients experienced minor complications: psychomotor agitation and QT prolongation, without causing major arrhythmias. Beta-blocker therapy was fully tolerated in all patients.

\section{Discussion}

Many anti-arrhythmic drugs are used in both acute and maintenance treatment of re-entry and automatic tachycardias in the first year of life; however, to date no consensus exists regarding the most effective drug, and there is still a wide variability in the treatments. According to the European guidelines, in case of re-entry tachycardias the first antiarrhythmic choice should be a Class 1A, Class $1 \mathrm{C}$ or Class 3 antiarrhythmic agent, except for amiodarone that should only be used if other drugs fail to control the arrhythmia. In case of automatic tachycardias, the first choice should be digoxin, with a Class $1 \mathrm{C}$ agent or beta-blocker added in case of failure, and using amiodarone as a third-line drug [4]. Our data demonstrate the effectiveness of flecainide and beta-blockers for a long-term follow up, starting from neonatal age up to childhood. In re-entry tachycardias we focused on the antiarrhythmic properties of oral flecainide as maintenance therapy. Flecainide, an IC anti-arrhythmic drug, has optimal pharmacodynamic properties, namely, the ability to slow the rate of diastolic depolarization plus it is highly effective in inducing anterograde and 
Table 1 Characteristics of the patients with re-entry tachycardias

\begin{tabular}{|c|c|c|c|c|c|c|c|}
\hline & Types of re-entry & Onset (days) & $\mathrm{CHD}$ & $\begin{array}{l}\text { Maximum HR } \\
(\mathrm{bpm})\end{array}$ & Symptoms & Maintenance therapy & $\begin{array}{l}\text { Time to } \\
\mathrm{CV} \text { (days) }\end{array}$ \\
\hline 1 & AVRT & 32 & & 300 & Minor & Flecainide + nadolol & 7 \\
\hline 2 & AVRT & Fetal & & 280 & Minor & Flecainide + nadolol & 15 \\
\hline 3 & AVRT & 1 & & 280 & Minor & Flecainide + sotalol & 8 \\
\hline 4 & AVRT & 64 & & 240 & Minor & Flecainide & 1 \\
\hline 5 & AVRT & 18 & & 300 & $\mathrm{HF}$ & Flecainide & 1 \\
\hline 6 & AVNRT & 1 & & 240 & Minor & Flecainide & 1 \\
\hline 7 & AVRT & 230 & & 300 & $\mathrm{HF}$ & Flecainide & 1 \\
\hline 8 & AVRT & 2 & & 270 & Minor & Flecainide & 1 \\
\hline 9 & AVRT & 12 & & 250 & Minor & Flecainide & 1 \\
\hline 10 & AVRT & 14 & & & Minor & Flecainide & 1 \\
\hline 11 & AVRT & 10 & & 280 & $\mathrm{HF}$ & Flecainide & 1 \\
\hline 12 & AVRT & 13 & & 320 & Shock & Flecainide & 1 \\
\hline 13 & AVRT & 1 & & 250 & $\mathrm{HF}$ & Amiodarone + nadolol & 22 \\
\hline 14 & AVRT & 1 & & 320 & Minor & Flecainide & 1 \\
\hline 15 & AVRT & 256 & & 300 & Minor & Flecainide + sotalol & 7 \\
\hline 16 & AVRT & 56 & & 260 & $\mathrm{HF}$ & Flecainide & 1 \\
\hline 17 & AVNRT & 6 & & 260 & Minor & Flecainide & 1 \\
\hline 18 & AVRT & 1 & & 261 & Minor & Flecainide + propranolol & 15 \\
\hline 19 & AVNRT & 13 & VSD & 215 & Minor & Flecainide & 1 \\
\hline 20 & AVRT & 15 & & & $\mathrm{HF}$ & Flecainide + digoxin & 7 \\
\hline 21 & AVRT & 26 & & 280 & & Flecainide + digoxin & 4 \\
\hline 22 & AVRT & 11 & & & & Flecainide + digoxin & 2 \\
\hline 23 & AVRT & Fetal & & & $\mathrm{HF}$ & Flecainide + sotalol & 15 \\
\hline 24 & AVRT & 10 & & 340 & $\mathrm{HF}$ & Flecainide & 1 \\
\hline 25 & AVRT & 36 & & 280 & Minor & Flecainide + nadolol & 3 \\
\hline 26 & AVRT & 72 & & 300 & $\mathrm{HF}$ & Flecainide & 1 \\
\hline 27 & AVRT & 27 & & 280 & Minor & Flecainide + digoxin & 4 \\
\hline 28 & AVRT & 8 & & 300 & Shock & Flecainide & $\mathrm{D}$ \\
\hline 29 & AVRT & 15 & & 260 & $\mathrm{HF}$ & Flecainide & 1 \\
\hline 30 & AVRT & Fetal & & 300 & $\mathrm{HF}$ & Flecainide + digoxin & 4 \\
\hline 31 & AVRT & 15 & Ebstein & 200 & $\mathrm{HF}$ & Flecainide + digoxin & 3 \\
\hline 32 & AVRT & 3 & & 300 & Minor & Flecainide & 1 \\
\hline 33 & AVRT & Fetal & & 220 & $\mathrm{HF}$ & Flecainide & 1 \\
\hline 34 & AVRT & 12 & $A S D+V S D$ & 260 & $\mathrm{HF}$ & Flecainide + propranolol & 7 \\
\hline 35 & AVRT & 1 & & 240 & Minor & Flecainide & 1 \\
\hline 36 & AVNRT & 12 & & 220 & HF & Flecainide & 1 \\
\hline 37 & AVRT & 224 & & 280 & Minor & Flecainide + nadolol & 6 \\
\hline 38 & AVRT & 21 & & 201 & HF & Flecainide & 1 \\
\hline 39 & AVRT & 24 & & 300 & $\mathrm{HF}$ & Flecainide + nadolol & 5 \\
\hline 40 & AVRT & 7 & & 300 & Minor & Flecainide + nadolol & 7 \\
\hline 41 & AVRT & 1 & & 280 & Minor & Flecainide & 1 \\
\hline 42 & AVRT & 36 & & 290 & HF & Amiodarone + nadolol & 15 \\
\hline 43 & AVRT & 11 & & 280 & Minor & Flecainide + nadolol & 7 \\
\hline 44 & AVRT & 1 & & 225 & Minor & Flecainide & 1 \\
\hline 45 & PJRT & Fetal & & 217 & $\mathrm{HF}$ & Flecainide + nadolol & 15 \\
\hline
\end{tabular}

AVRT atrioventricular re-entry tachycardia, CV cardioversion, $A V N R T$ atrioventricular nodal re-entry tachycardia, PJRT paroxysmal junctional re-entry tachycardia, ASD atrial septal defect, $C H D$ congenital heart diseases, D death, Ebstein Ebstein's anomaly, HF heart failure, HR heart rates, minor minor symptoms, NCLV non-compacted left ventricular cardiomyopathy, shock cardiogenic shock, VSD ventricular septal defect 
Table 2 Characteristics of the patients with automatic tachycardias

\begin{tabular}{|c|c|c|c|c|c|c|}
\hline & Onset (days) & $\mathrm{CHD}$ & Maximum HR (bpm) & Symptoms & Maintenance therapy & $\begin{array}{l}\text { Time to } \\
\text { CV (days) }\end{array}$ \\
\hline 1 & Fetal & & 240 & Minor & Flecainide + nadolol & 40 \\
\hline 2 & Fetal & & 220 & Minor & Flecainide + sotalol & 22 \\
\hline 3 & 285 & & 280 & Minor & Flecainide + sotalol & 20 \\
\hline 4 & 2 & $\mathrm{VSD}+\mathrm{NCLV}$ & 210 & Minor & Amiodarone + nadolol & 40 \\
\hline 5 & 303 & & 300 & Shock & Flecainide + nadolol & 23 \\
\hline 6 & 36 & VSD & 180 & HF & Flecainide + nadolol & 22 \\
\hline 7 & 1 & & 240 & $\mathrm{HF}$ & Flecainide + sotalol & 32 \\
\hline 8 & 13 & & 230 & Minor & Nadolol & 6 \\
\hline 9 & 1 & & 195 & Minor & Propranolol & 26 \\
\hline 10 & 6 & VSD & 250 & Minor & Sotalol & 10 \\
\hline
\end{tabular}

CHD congenital heart diseases, CV cardioversion, HF heart failure, $H R$ heart rates, minor minor symptoms, NCLV non-compacted left ventricular, VSD ventricular septal defect

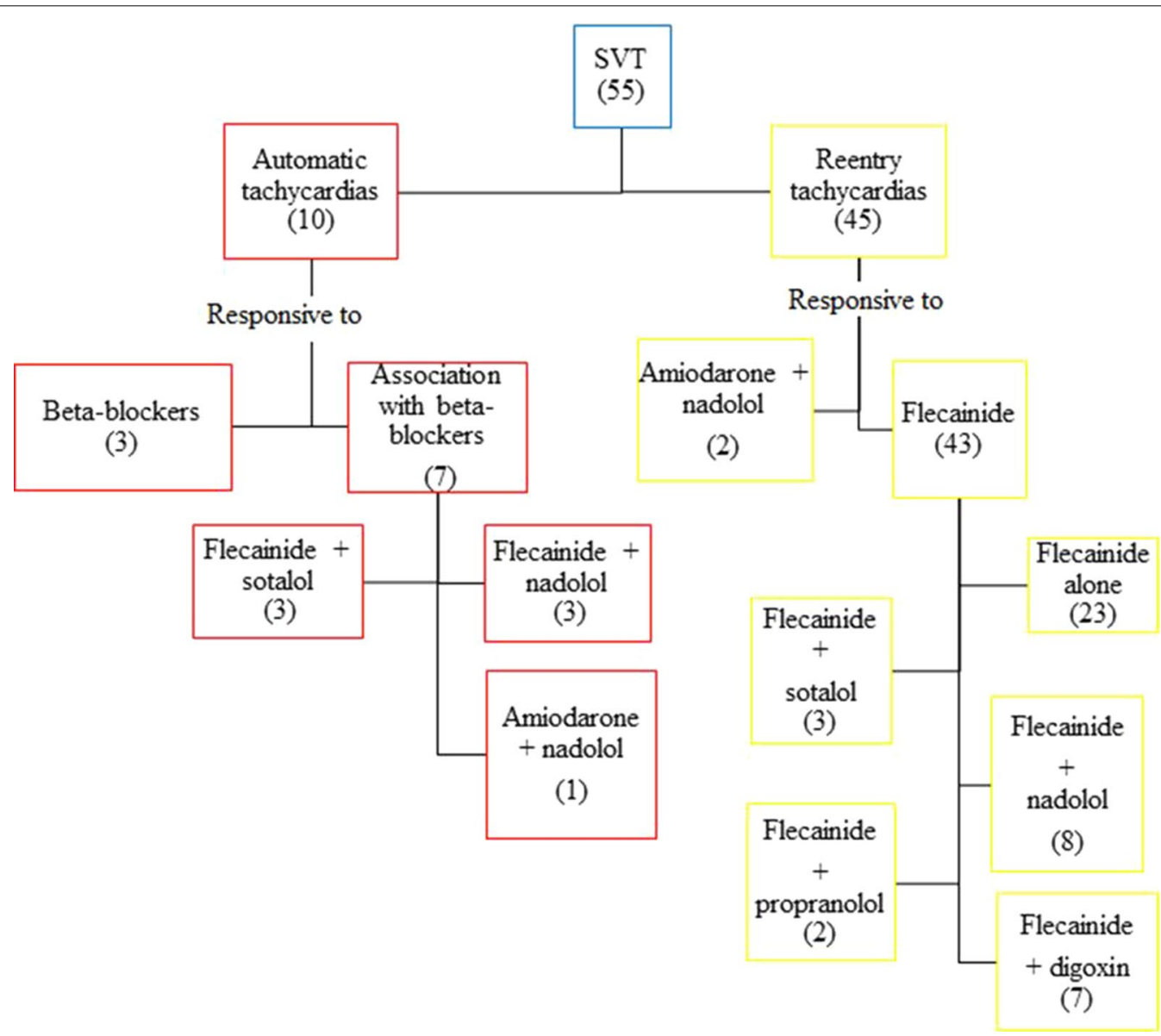

Fig. 1 Flow chart of responses

retrograde conduction block on accessory pathways. It also presents good pharmacokinetic properties, including relatively fast oral absorption and a long half-life elimination of about 11-12 h $[13,14]$. Thanks to these properties, this drug showed high efficacy in $73-100 \%$ of supraventricular tachycardias [15]. Unlike in adulthood, 
Table 3 Time to cardioversion (days)

\begin{tabular}{llll}
\hline & $\begin{array}{l}\text { Re-entry } \\
\text { tachycardias }\end{array}$ & Automatic tachycardias & \\
\hline $\begin{array}{l}\text { Mean } \pm \text { SD } \\
\text { (days) }\end{array}$ & $4.5 \pm 5.2$ & $24.1 \pm 11.1$ & $p<0.05$ \\
Median (days) & $1(1$ to 22$)$ & $22.5(6$ to 40$)$ & \\
\hline
\end{tabular}

where a significant incidence of pro-arrhythmic effects was reported in the CAST study, flecainide-induced arrhythmias were documented in less than $7 \%$ of the paediatric population $[15,16]$. Propafenone, another IC anti-arrhythmic drug, demonstrated good results in clinical trials [17]. In a prospective study comparing four anti-arrhythmic drugs in long-term prophylaxis of SVT, it appeared to be less effective than flecainide [10]. Few studies are reported with sotalol, a Class III anti-arrhythmic agent with beta-blocking properties; although high effectiveness has been reported with elevated doses in paediatric SVTs, we decided to use it in combination with flecainide, given the encouraging results of this combination [18]. Beta-blockers in monotherapy might be an alternative choice, both in re-entry and automatic tachycardias. In the SAMIS trial, one of the first prospective studies in infants, long-term treatment with propranolol in monotherapy demonstrated non-inferiority when compared with digoxin in monotherapy [19]. In automatic atrial tachycardia, beta-blockers were used in monotherapy or combined mostly with digoxin or flecainide [20]. Finally, amiodarone, a third-class antiarrhythmic agent, can only be considered an alternative for infants with resistant supraventricular tachycardias due to its well-known adverse events: in a recent cohort of 150 paediatric patients receiving amiodarone in acute and long-term treatment, $50.8 \%$ developed thyroid dysfunction, especially the newborns (66.7\%) [12]. Furthermore, a recent comparative study between flecainide and amiodarone for the treatment of paediatric supraventricular tachycardias demonstrated the non-inferiority of the former, both in patients with congenital heart disease and those with structurally normal hearts. Lastly, in all the patients in whom amiodarone was ineffective, the shift to flecainide allowed for controlling the arrhythmia [11]. Perry et al. [14] observed 45 patients with supraventricular tachycardias (both re-entries and automatics) and showed that flecainide controlled about $81 \%$ of cases. O'Sullivan et al. [21] reported that 39 infants with sustained atrioventricular re-entry tachycardias nonresponsive to digoxin were treated with flecainide, with about $96 \%$ of success. Ferlini et al. [9] showed that neonates could be treated with this drug both in acute and mid-term therapy with an effectiveness of approximately
$85 \%$. Our data showed an efficacy of about $51.1 \%$ in reentry tachycardias as monotherapy; however, when we combined it with beta-blockers or digoxin, a stable cardioversion was achieved in up to $95.5 \%$ of cases. Digoxin has been reported to be successful when combined with flecainide both in fetal and neonatal supraventricular tachycardias [20,22]. The effectiveness of combined treatment compared to monotherapy may be related to the enhanced adrenergic tone of the neonate, representing a trigger for re-entry arrhythmias. In order to achieve stable rhythm control, automatic tachycardias, known to be more resistant to incessant antiarrhythmic treatment, require a pharmacological combination more frequently than re-entry forms [20].

Price et al. [23] considered 10 patients with supraventricular tachycardias (eight re-entry and two automatic) in the first year of life who were unresponsive to at least two anti-arrhythmic agents: the combination of flecainide and sotalol was effective in all patients in an average of twelve days. Also in our experience, although sotalol is scarcely effective in monotherapy, when combined with flecainide, even at beta-blocking doses, it was able to obtain a stable cardioversion in resistant forms without prolonging the QTc interval [24]. Von Alvensleben et al. [25] considered 28 patients with supraventricular tachycardias (27 with re-entries and 1 with focal atrial tachycardia) under 2 years of age; nadolol alone resolved arrhythmia in 20/28 (71.4\%) cases, and for the other six this result was achieved when combined with flecainide. Perry et al. [14] obtained stable cardioversion with flecainide alone for $9 / 13$ (69.2\%) patients with ectopic atrial tachycardias. In our study, beta-blockers proved to be effective in monotherapy in a minority (30\%), but again, the combination with flecainide allowed for controlling almost all resistant tachycardias. Therefore, a substantial number of infants with re-entry supraventricular tachycardias and the majority with automatic forms, required the combination of two antiarrhythmic agents for a stable cardioversion. Flecainide and nadolol or sotalol seemed to be the most effective choice. The time to sustained sinus rhythm was significantly longer in automatic tachycardias than in re-entry forms $[20,26]$. In one case, after obtaining a satisfactory rate control, we discharged the patient, and in the end observed stable conversion to sinus rhythm in all patients. In two cases a stable cardioversion was obtained when anti-arrhythmic drugs were administered more frequently than two or three times a day, with the strict recommendation to monitor ECG and blood flecainide concentration continuously in order to prevent toxicity $[5,27]$. This different administration rate may be related to the variability of the drug's pharmacokinetics, often reported in infants $[14,15]$. Measuring flecainide blood concentration helped us to quantify 
the correct doses of the drug. Therefore, we strongly recommend this approach in clinical practice when possible in order to avoid life-threatening complications due to overdosage.

In line with our results, we have proposed a protocol for the treatment of infant supraventricular tachycardias, differentiating re-entry from automatic atrial tachycardias (Fig. 2). With regard to beta-blockers we decided to prefer nadolol over propranolol thanks to its longer halflife, and because of the excellent clinical results recently reported in maintenance therapy of SVT [25].

With flecainide treatment we observed two major ventricular arrhythmias. As for the patient coming from another hospital who died, flecainide toxicity could indeed have been the cause of death, possibly owing to a poor metabolizing mechanism, as also potentially reported in a recent Japanese study [28]. Incessant supraventricular tachyarrhythmias, ventricular tachycardias and severe bradycardias have been reported during flecainide treatment, therefore it is recommended to start therapy during hospitalization and to closely monitor the ECG and plasma concentration [15, 29]. Pro-arrhythmic

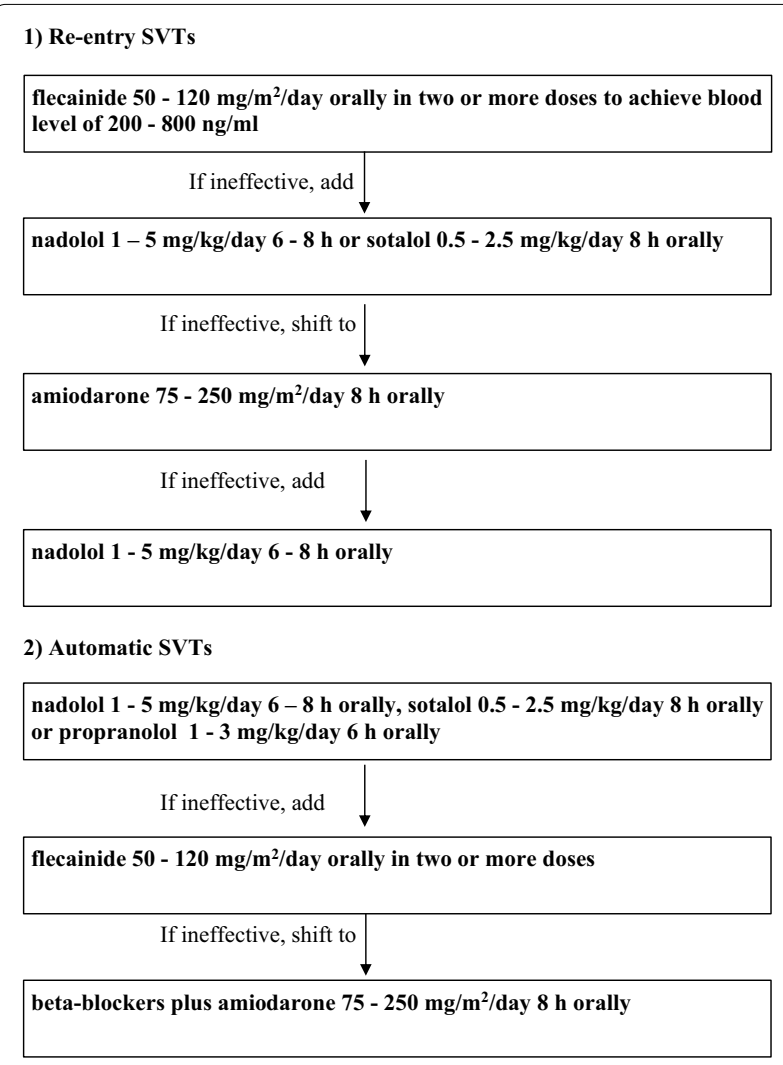

Fig. 2 Protocol proposal for the treatment of supraventricular tachycardias in the first year of life effects and cardiac arrest seem to be more common among patients with underlying heart diseases or impaired systemic ventricular function than in those with a normal heart [30]. However, in a recent study, a comparison between the use of flecainide and other drugs in patients with cardiomyopathy or structural heart diseases showed that there were no differences in the incidence of cardiac arrest or death [31].

In our work, the gestational age at birth, birth weight and perinatal age were not predisposing factors influencing a different antiarrhythmic approach. Moreover, the efficacy of antiarrhythmic agents was not influenced by the presence of CHD defects, most of which were well-tolerated.

\section{Conclusions}

Our experience shows that in re-entry SVTs, flecainide may be an effective treatment option as monotherapy; nonetheless, better results can be achieved in combination with beta-blockers. In automatic tachycardias, the association of flecainide with beta-blockers (nadolol or sotalol) seems highly effective in achieving heart rate control therapy with the aim of delayed cardioversion. In specific cases, tailored management could be required in order to maintain stable flecainide blood levels and obtain better control of arrhythmia, i.e. by increasing the number of administrations per day. It is imperative to remember that this drug should always be used with caution due to the percentage of adverse events: the possibility of monitoring the blood levels of flecainide may be helpful for this purpose.

\section{Study limitations}

The main limitation of this study was its retrospective form, due to several changes in the clinical management of the patients' therapy which did not allow for planning a prospective study. Moreover, the relatively small cohort of patients attenuated the statistical significance of our findings. In addition, the delineation of shared protocols concerning acute and long-term anti-arrhythmic treatment is advocated. For this purpose, more data from randomized controlled trials are needed.

\section{Abbreviations \\ AVRT: Atrioventricular re-entry tachycardia; AVNRT: Atrioventricular nodal re- entry tachycardia; CHD: Congenital heart disease; PJRT: Paroxysmal junctional reciprocating tachycardia; SVT: Supraventricular tachycardia.}

\section{Acknowledgements}

Not applicable.

\section{Authors' contributions}

LDS conceptualized, designed the work, and reviewed the manuscript. GC designed the work, drafted the initial manuscript, and reviewed the 
manuscript. GB, MG, GR, AB, FV, SF and GP reviewed the manuscript. All authors read and approved the final manuscript.

\section{Funding}

This research received no specific grants from any funding agency, commercial or not-for-profit sectors.

\section{Availability of data and materials}

The data generated by and used in the study is available from the corresponding author upon reasonable request.

\section{Ethics approval and consent to participate}

The study protocol (entitled 001_TPSV) was approved by the ethics committee of the Anna Meyer Children's University Hospital (Florence). We obtained written informed consent from all the parents of participants in the study. All the procedures were performed in accordance with the Helsinki Declaration.

\section{Consent for publication}

Not applicable.

\section{Competing interests}

The authors declare that they have no competing interests.

\section{Author details}

${ }^{1}$ Department of Health Sciences, Post-Graduate School of Paediatrics, Anna Meyer Children's University Hospital, Florence, Italy. ${ }^{2}$ Neonatology Department and Neonatal Intensive Care Unit, Careggi University Hospital, Florence, Italy. ${ }^{3}$ Cardiology Unit, Anna Meyer Children's University Hospital, Viale Gaetano Pieraccini 24, 50139 Florence, Italy.

Received: 17 June 2020 Accepted: 27 December 2020 Published online: 15 March 2021

\section{References}

1. Chu PY, Hill KD, Clark RH, Brian Smith P, Hornik CP. Treatment of supraventricular tachycardia in infants: analysis of a large multicenter database. Early Hum Dev. 2015;91(6):345-50.

2. Vignati G, Balla E, Mauri L, Lunati M, Figini A. Clinical and electrophysiologic evolution of the Wolff-Parkinson-White syndrome in children: impact on approaches to management. Cardiol Young. 2000;10(4):367-75.

3. Pickoff AS, Singh S, Flinn CJ, McCormack J, Stolfi A, Gelband H. Atrial vulnerability in the immature canine heart. Am J Cardiol. 1985;55(11):1402-6.

4. Brugada J, Blom N, Sarquella-Brugada G, Blomstrom-Lundqvist C, Deanfield J, Janousek J, et al. Pharmacological and non-pharmacological therapy for arrhythmias in the pediatric population: EHRA and AEPCArrhythmia Working Group joint consensus statement. Europace. 2013:15:1337-82

5. Ludomirky A, Garson A. Supraventricular tachycardia . In: Garson A Jr, Bricker JT, McNamara DG, editors. The science and practice of pediatric cardiology. Philadelphia: Lea \& Febiger; 1990. p. 1809-48.

6. Schwartz PJ, Garson A, Paul T, Stramba-Badiale M, Vetter VL, Villain E, et al. Guidelines for the interpretation of the neonatal electrocardiogram: a Task Force of the European Society of Cardiology. Eur Heart J. 2002;23(17):1329-44.

7. Vignati G, Annoni G. Characterization of supraventricular tachycardia in infants: clinical and instrumental diagnosis. Curr Pharm Des. 2008;14:729-35.

8. Buttà C, Tuttolomondo A, Giarrusso L, Pinto A. Electrocardiographic diagnosis of atrial tachycardia: classification, P-wave morphology, and differential diagnosis with other supraventricular tachycardias. Ann Noninvasive Electrocardiol. 2015:20:314-27.

9. Ferlini M, Colli AM, Bonanomi C, Salvini L, Galli MA, Salice P, et al. Flecainide as first-line treatment for supraventricular tachycardia in newborns. J Cardiovasc Med. 2009;10(5):372-5.

10. Drago F, Silvetti MS, De Santis A, Marcora S, Fazio G, Anaclerio S, et al. Paroxysmal reciprocating supraventricular tachycardia in infants: Electrophysiologically guided medical treatment and long-term evolution of the re-entry circuit. Europace. 2008;10:629-35.
11. Hill AC, Silka MJ, Bar-Cohen Y. A comparison of oral flecainide and amiodarone for the treatment of recurrent supraventricular tachycardia in children. PACE Pacing Clin Electrophysiol. 2019:42:670-7.

12. Creo A, Anderson H, Cannon B, Lteif A, Kumar S, Tebben P, et al. Patterns of amiodarone-induced thyroid dysfunction in infants and children. Heart Rhythm. 2019;16:1436-42.

13. Crozier I. Flecainide in the Wolff-Parkinson-White syndrome. Am J Cardiol. 1992;70:A26-32

14. Perry JC, McQuinn RL, Smith RT, Gothing C, Fredell P, Garson A. Flecainide acetate for resistant arrhythmias in the young: efficacy and pharmacokinetics. J Am Coll Cardiol. 1989;14(1):185-91.

15. Perry JC, Garson A. Flecainide acetate for treatment of tachyarrhythmias in children: review of world literature on efficacy, safety, and dosing. Am Heart J. 1992:124:1622-4

16. Investigators CAST. Effect of encainide and flecainide on mortality in a randomised trial of arrhythmia supression after myocardial infarction. $\mathrm{N}$ Engl J Med. 1989;321:406-12.

17. Vignati $G$, Mauri L, Figini A. The use of propafenone in the treatment of tachyarrhythmias in children. Eur Heart J. 1993;14:546-50.

18. Knudson JD, Cannon BC, Kim JJ, Moffett BS. High-dose sotalol is safe and effective in neonates and infants with refractory supraventricular tachyarrhythmias. Pediatr Cardiol. 2011;32:896-903.

19. Sanatani S, Potts JE, Reed JH, Saul JP, Stephenson EA, Gibbs KA, et al. The study of antiarrhythmic medications in infancy (SAMIS): a multicenter, randomized controlled trial comparing the efficacy and safety of digoxin versus propranolol for prophylaxis of supraventricular tachycardia in infants. Circ Arrhythmia Electrophysiol. 2012;5:984-91.

20. Salerno JC, Kertesz NJ, Friedman RA, Fenrich AL. Clinical course of atrial ectopic tachycardia is age-dependent: results and treatment in children $<3$ or $\geq 3$ years of age. J Am Coll Cardiol. 2004;43:438-44.

21. O'Sullivan JJ, Gardiner HM, Wren C. Digoxin or flecainide for prophylaxis of supraventricular tachycardia in infants? J Am Coll Cardiol. 1995:26(4):991-4.

22. Miyoshi T, Maeno Y, Hamasaki T, Inamura N, Yasukochi S, Kawataki M, et al. Antenatal therapy for fetal supraventricular tachyarrhythmias: multicenter trial. J Am Coll Cardiol. 2019:74:874-85.

23. Price JF, Kertesz NJ, Snyder CS, Friedman RA, Fenrich AL. Flecainide and sotalol: a new combination therapy for refractory supraventricular tachycardia in children <1 year of age. J Am Coll Cardiol. 2002;39:517-20.

24. Saul JP, Ross B, Schaffer MS, Beerman L, Melikian AP, Shi J, et al. Pharmacokinetics and pharmacodynamics of sotalol in a pediatric population with supraventricular and ventricular tachyarrhythmia. Clin Pharmacol Ther. 2001;69:145-57

25. von Alvensleben JC, LaPage MJ, Caruthers R, Bradley DJ. Nadolol for treatment of supraventricular tachycardia in infants and young children. Pediatr Cardiol. 2017;38:525-30

26. Mehta AV, Sanchez GR, Sacks EJ, Casta A, Dunn JM, Donner RM. Ectopic automatic atrial tachycardia in children: clinical characteristics, management and follow-up. J Am Coll Cardiol. 1988:11:379-85.

27. Belli G, Giovannini M, Porcedda G, Moroni M, la Marca G, Capponi G, et al. Incessant automatic atrial tachycardia in a neonate successfully treated with nadolol and closely spaced doses of flecainide: a case report. Pediatr Rep. 2020:12(3):108-13.

28. Horie A, Ishida K, Shibata K, Taguchi M, Ozawa A, Hirono K, et al. Pharmacokinetic variability of flecainide in younger Japanese patients and mechanisms for renal excretion and intestinal absorption. Biopharm Drug Dispos. 2014;35(3):145-53.

29. Tavera MC, Bassareo PP, Neroni P, Follese C, Manca D, Montis S, et al. Supraventricular tachycardia in neonates: antiarrhythmic drug choice dilemma. J Matern Neonatal Med. 2011:24(3):541-4.

30. Fish FA, Gillette PC, Benson DW. Proarrhythmia, cardiac arrest and death in young patients receiving encainide and flecainide. J Am Coll Cardiol. 1991;18(2):356-65.

31. Moffett BS, Valdes SO, Lupo PJ, Miyake C, Krenek M, , et al. Flecainide use in children with cardiomyopathy or structural heart disease. Pediatr Cardiol. 2015;36(1):146-50.

\section{Publisher's Note}

Springer Nature remains neutral with regard to jurisdictional claims in published maps and institutional affiliations. 